PROCEEDINGS OF THE

AMERICAN MATHEMATICAL SOCIETY

Volume 125, Number 4, April 1997, Pages 1105-1109

S 0002-9939(97)03680-0

\title{
BEST APPROACH REGIONS FOR POTENTIAL SPACES
}

\author{
JOSÉ A. RAPOSO AND JAVIER SORIA
}

(Communicated by J. Marshall Ash)

\begin{abstract}
We characterize the approach regions so that the non-tangential maximal function is of weak-type on potential spaces, for which we use a simple argument involving Carleson measure estimates.
\end{abstract}

\section{INTRODUCTION}

The classical theorem of Fatou, about the non-tangential convergence of the harmonic extension of functions in $L^{p}\left(\mathbb{R}^{n}\right)$, has been widely studied in the recent years. Nagel and Stein ([NS]) have found a characterization of those regions for which Fatou's theorem remains valid. As Littlewood showed $([\mathrm{Li}])$, convergence on tangential directions fails for some functions in $L^{p}\left(\mathbb{R}^{n}\right)$. However, Nagel, Rudin and Shapiro $([\mathrm{NRS}])$ were able to show that if one considers functions in some particular subspaces (potential spaces), with an appropriate regularity, then it is possible to find a tangential approach region, so that Fatou's theorem holds true.

The main result we prove below is in the spirit of [NS]; namely, we find a characterization of the approach regions for which the associated maximal operator is of weak-type, with respect to the spaces considered in [NRS]. The condition that we obtain (Theorem 2.6(b)) says that the measure of the cross section at level $t$ of the region is of the order of $r(t)^{n}$, where $r$ is determined by the potential space. In particular, when we deal with $L^{p}\left(\mathbb{R}^{n}\right)$, then $r(t) \approx t$ and we obtain the main theorem of $[\mathrm{NS}]$. As a consequence, we give an example of such a region, not contained in any of those considered in [NRS].

The techniques we use in the proof of the theorem are based on the work of Andersson and Carlsson $([\mathrm{AC}])$, for the case of non-tangential approach regions, and an extension of the notion of Carleson measure to general domains ([CS]).

Let us first set up some of the definitions we need in the sequel (see also [CS]).

Definition 1.1. - An open set $\Omega \subset \mathbb{R}_{+}^{n+1}$ is an approach region if $0 \in \bar{\Omega}$. For $x \in \mathbb{R}^{n}$ we set $\Omega_{x}=x+\Omega$.

- If $P$ is the Poisson kernel in $\mathbb{R}^{n}, P_{t}(y)=t^{-n} P\left(t^{-1} y\right)$ and $f \in L^{p}\left(\mathbb{R}^{n}\right)$, the maximal operator associated to $\Omega$ is

$$
N_{\Omega} f(x)=\sup _{(y, t) \in \Omega_{x}}\left|f * P_{t}(y)\right| .
$$

Received by the editors August 9, 1994 and, in revised form, October 4, 1995.

1991 Mathematics Subject Classification. Primary 42B25, 42B20.

Key words and phrases. Weak-type inequalities, maximal functions, approach regions.

This work has been partially supported by DGICYT PB94-0879.

(C)1997 American Mathematical Society 
For the following set of definitions we will consider a function $r: \mathbb{R}^{+} \longrightarrow \mathbb{R}^{+}$. Later on, this function will be chosen in a particular way, satisfying some extra conditions.

Definition 1.2. Fix $r: \mathbb{R}^{+} \longrightarrow \mathbb{R}^{+}$.

$-\Gamma_{r}(x)=\{(y, t) ;|x-y|<r(t)\}$.

- Given a set $E \subset \mathbb{R}^{n}$, we define the $r$-tent,

$$
T_{r}(E)=\mathbb{R}_{+}^{n+1} \backslash \bigcup_{x \notin E} \Gamma_{r}(x) .
$$

- Given an outer measure $\mu$ in $\mathbb{R}_{+}^{n+1}$, we say that it is an $r$-Carleson measure if there exists a constant $C>0$ such that, for every $(x, t) \in \mathbb{R}_{+}^{n+1}$,

$$
\mu\left(T_{r}(B(x, t))\right) \leq C t^{n} .
$$

Remark 1.3. If $r(t)=t$, we deal with the classical situation of cones, tents and Carleson measures. Also, we could have chosen cubes instead of balls in the definition of $r$-Carleson measures.

There are some equivalent ways to define these tents.

Proposition 1.4. (a) $T_{r}(E)=\{(y, t) ; B(y, r(t)) \subset E\}=\left\{(y, t) ; \mathrm{d}\left(y, \mathbb{R}^{n} \backslash E\right) \geq\right.$ $r(t)\}$.

(b) $T_{r}(B(x, t))=x+T_{r}(B(0, t))$.

Proof. (a) can be proved as in Lemma 5(iii) of [CS] and (b) is easy.

In order to obtain estimates for the maximal operator, we need to consider arbitrary open sets. An important example is given by the following result.

Proposition 1.5. If $\mu$ is an $r$-Carleson measure, then there exists $C>0$ such that for all open sets $G \subset \mathbb{R}^{n}, \mu\left(T_{r}(G)\right) \leq C|G|$.

Proof. Let $G \subset \mathbb{R}^{n}$ be an open set. We find a Whitney decomposition of $G$ (see $[\mathrm{St}]$ ), given by a sequence of cubes $\left\{Q_{k}\right\}_{k}$ with disjoint interior so that $G=\bigcup_{k} Q_{k}$, and $\operatorname{diam}\left(Q_{k}\right) \approx \mathrm{d}\left(Q_{k}, \mathbb{R}^{n} \backslash G\right)$. Now, it suffices to show that there exists a constant $C=C(n)>0$ so that if $Q_{k}^{*}=C Q_{k}$, then

$$
T_{r}(G) \subset \bigcup_{k} T_{r}\left(Q_{k}^{*}\right) .
$$

Choose $(y, t) \in T_{r}(G)$. Then $\mathrm{d}\left(y, \mathbb{R}^{n} \backslash G\right) \geq r(t)$. Let $k \in \mathbb{Z}$ so that $y \in Q_{k}$. We want to prove that $(y, t) \in T_{r}\left(Q_{k}^{*}\right)$, which is equivalent (by Proposition 1.4) to showing that $B(y, r(t)) \subset Q_{k}^{*}$. Let $x_{k}$ be the center of $Q_{k}$. If $|z-y|<r(t)$, then $\left|z-x_{k}\right|<r(t)+\operatorname{diam}\left(Q_{k}\right) / 2$. Therefore, it all reduces to finding $C$, so that $r(t) \leq C \operatorname{diam}\left(Q_{k}\right)$. Let $u \notin G$ and $x \in Q_{k}$ :

$$
r(t) \leq \mathrm{d}\left(y, \mathbb{R}^{n} \backslash G\right) \leq|y-x|+|x-u| \leq \operatorname{diam}\left(Q_{k}\right)+|x-u| .
$$

Taking the infimum on $u$ and $x$, we finally obtain

$$
r(t) \leq \operatorname{diam}\left(Q_{k}\right)+\mathrm{d}\left(Q_{k}, \mathbb{R}^{n} \backslash G\right) \leq C \operatorname{diam}\left(Q_{k}\right) .
$$




\section{BEST APPROACH REGIONS}

We now give the characterization of those approach regions for which the maximal operator $N_{\Omega}$ is of weak-type on a certain space. It is very interesting to observe that the boundedness properties of this operator are determined by the geometry of the regions. We make this clearer by introducing the following sets.

Definition 2.1. Let $\Omega \subset \mathbb{R}_{+}^{n+1}$ be an approach region and $r: \mathbb{R}^{+} \longrightarrow \mathbb{R}^{+}$. We define,

- $\Omega_{x}(t)=\left\{y ;(y, t) \in \Omega_{x}\right\}$.

- $S_{r}(x, t)=\left\{y ; \Omega_{y} \cap T_{r}(B(x, t)) \neq \emptyset\right\}$.

- $\mu_{\Omega}(E)=\left|\left\{x ; \Omega_{x} \cap E \neq \emptyset\right\}\right|, E \subset \mathbb{R}_{+}^{n+1}$.

As in [NRS], we introduce the following potential space (associated now to a finite measure).

Definition 2.2. Let $\nu$ be a positive finite measure in $\mathbb{R}^{n}$. For $1 \leq p<\infty$ we define

$$
L_{\nu}^{p}\left(\mathbb{R}^{n}\right)=\left\{f ; f=F * d \nu, F \in L^{p}\left(\mathbb{R}^{n}\right)\right\},
$$

endowed with the quotient norm $\|f\|_{L_{\nu}^{p}}=\inf \left\{\|F\|_{p} ; f=F * d \nu\right\}$. We will denote by $V(x, t)=V_{t}(x)=P_{t} * d \nu(x)$, the harmonic extension of $\nu$.

Remark 2.3. Some interesting examples are given when $d \nu(x)=G_{\alpha}(x) d x$, where $G_{\alpha}$ is the Bessel potential (see [St]). Observe also that if $\nu=\delta$, the Dirac delta, then $L_{\nu}^{p}=L^{p}$.

Definition 2.4. Let $\Omega$ be an approach region and $r: \mathbb{R}^{+} \longrightarrow \mathbb{R}^{+}$. We say that $\Omega$ satisfies the $r$ condition if $\Gamma_{r}(x, t) \subset \Omega$, for all $(x, t) \in \Omega$, where $\Gamma_{r}(x, t)=$ $\{(y, s) ;|y-x|<r(s)-r(t)\}$.

Observe that if $r(t)=t$, this is equivalent to the cone condition assumed in [NS]. The following result gives very precise information on the region $\Gamma_{r}$ determined by a measure $\nu$. The proof is easy and we will omit it (see also [NRS]).

Proposition 2.5. Let $\nu$ be a positive finite measure in $\mathbb{R}^{n}$ and let $1 \leq p<\infty$. Set $r(t)=\left\|V_{t}\right\|_{p^{\prime}}^{-p / n}$. Then, $r$ is a continuous increasing function, $0<r(t)<\infty, t>0$ and $t^{-1} r(t) \longrightarrow\left(\|\nu\|\|P\|_{p^{\prime}}\right)^{-p / n}$ as $t \longrightarrow \infty$. Moreover, if $d \nu(x)=k(x) d x$, with $k \in L^{1} \backslash L^{p^{\prime}}$, then $r(t) \longrightarrow 0$ as $t \longrightarrow 0$ (and hence $r$ is onto) and $r(t) / t \longrightarrow \infty$ as $t \longrightarrow 0$. Also, if $\nu$ is an atomic measure, then $r(t) \approx t$, for $t>0$.

We now give the proof of our main result. We will restrict the hypothesis to a particular choice of $\nu$ (see Remark 2.7 for some extensions).

Theorem 2.6. Let $k \in L^{1}\left(\mathbb{R}^{n}\right)$ be a positive radially decreasing kernel and let $1 \leq p<\infty$. Assume $k \notin L^{p^{\prime}}\left(\mathbb{R}^{n}\right)$. Set $d \nu(x)=k(x) d x$ and $r(t)=\left\|V_{t}\right\|_{p^{\prime}}^{-p / n}$. Let $\Omega$ be an approach region satisfying the $r$ condition. Then, the following are equivalent:

(a) $N_{\Omega}: L_{\nu}^{p}\left(\mathbb{R}^{n}\right) \longrightarrow L^{p, \infty}\left(\mathbb{R}^{n}\right)$ is bounded.

(b) There exists $C>0$ such that, for all $t>0,|\Omega(t)| \leq C r(t)^{n}$.

(c) There exists $C>0$ such that, for all $(x, t) \in \mathbb{R}_{+}^{n+1},\left|S_{r}(x, t)\right| \leq C t^{n}$.

(d) $\mu_{\Omega}$ is an $r$-Carleson measure.

(e) There exists $C>0$ such that, for all $f \in L_{\nu}^{p}\left(\mathbb{R}^{n}\right)$ and $\lambda>0$,

$$
\left|\left\{x ; N_{\Omega} f(x)>\lambda\right\}\right| \leq C\left|\left\{x ; N_{\Gamma_{r}} f(x)>\lambda\right\}\right| .
$$


Proof. $(a) \Rightarrow(b)$. Fix $t>0$. Since

$$
\left\|V_{t}\right\|_{p^{\prime}}=\sup _{\|F\|_{p}=1}\left|\int_{\mathbb{R}^{n}} V_{t}(x) F(-x) d x\right|=\sup _{\|F\|_{p}=1}\left|V_{t} * F(0)\right|,
$$

we can find $F \in L^{p},\|F\|_{p}=1$ such that $\lambda \equiv\left|V_{t} * F(0)\right| \geq\left\|V_{t}\right\|_{p^{\prime}} / 2$. Let $x \in \mathbb{R}^{n}$ so that $(0, t) \in \Omega_{x}$ and $f=F * d \nu$. Then $|\Omega(t)|=\left|\left\{y ;(0, t) \in \Omega_{y}\right\}\right|$ and

$$
N_{\Omega} f(x)=\sup _{(y, s) \in \Omega_{x}}\left|V_{s} * F(y)\right| \geq\left|V_{t} * F(0)\right|=\lambda .
$$

Hence, $x \in\left\{N_{\Omega} f>\lambda / 2\right\}$ and

$$
|\Omega(t)| \leq\left|\left\{N_{\Omega} f>\lambda / 2\right\}\right| \leq \frac{C}{\lambda^{p}}\|f\|_{L_{\nu}^{p}}^{p} \leq C r(t)^{n} .
$$

$(b) \Rightarrow(c)$. Let us first show that for $(x, t) \in \mathbb{R}_{+}^{n+1}, S_{r}(x, r(t))=\left\{y ;(x, t) \in \Omega_{y}\right\}$. In fact, if $(x, t) \in \Omega_{y}$, then $\Omega_{y} \cap T_{r}(B(x, r(t))) \neq \emptyset$, and by definition this implies $y \in S_{r}(x, r(t))$. Conversely, if $\Omega_{y} \cap T_{r}(B(x, r(t))) \neq \emptyset$, there exists $(z, s) \in \Omega_{y}$ so that $B(z, r(s)) \subset B(x, r(t))$. Thus, we can find $z^{\prime} \in \Omega_{y}(s)$ with $\left|z^{\prime}-x\right|<r(t)-r(s)$, and since $\Omega$ satisfies the $r$ condition, $(x, t) \in \Omega_{y}$.

Thus, we easily obtain that $\left|S_{r}(x, r(t))\right|=|\Omega(t)|$, and by Proposition 2.5, given $t>0$ there exists $t^{\prime}>0$ so that $r\left(t^{\prime}\right)=t$. Hence,

$$
\left|S_{r}(x, t)\right|=\left|S_{r}\left(x, r\left(t^{\prime}\right)\right)\right|=\left|\Omega\left(t^{\prime}\right)\right| \leq C r\left(t^{\prime}\right)^{n}=C t^{n} .
$$

$(c) \Rightarrow(d)$. It is clear that $\mu_{\Omega}$ is an outer measure. Now, given $(x, t) \in \mathbb{R}_{+}^{n+1}$,

$$
\mu_{\Omega}\left(T_{r}(B(x, t))\right)=\left|\left\{y ; \Omega_{y} \cap T_{r}(B(x, t)) \neq \emptyset\right\}\right|=\left|S_{r}(x, t)\right| \leq C t^{n} .
$$

$(d) \Rightarrow(e)$. Since $\left\{x ; N_{\Gamma_{r}} f(x)>\lambda\right\}$ is an open set and

$$
\left|\left\{x ; N_{\Omega} f(x)>\lambda\right\}\right|=\mu_{\Omega}\left(\left\{(y, t) \in \mathbb{R}_{+}^{n+1} ;\left|f * P_{t}(y)\right|>\lambda\right\}\right),
$$

$(e)$ is a consequence of the fact that

$$
\left\{(y, t) \in \mathbb{R}_{+}^{n+1} ;\left|f * P_{t}(y)\right|>\lambda\right\} \subset T_{r}\left(\left\{x ; N_{\Gamma_{r}} f(x)>\lambda\right\}\right)
$$

(see [CS]) and Proposition 1.5.

$(e) \Rightarrow(a)$. This is immediate since $N_{\Gamma_{r}}$ is always a bounded operator (see [NRS]).

Remark 2.7. (i) In Theorem 2.6 one can still give partial results with weaker conditions. In fact, it suffices to observe that $(a) \Rightarrow(b)$ holds for any measure $\nu$ and any region $\Omega$. In $(b) \Rightarrow(c)$, the only condition we need on $r$ is that $r(0)=0$. For $(c) \Rightarrow(d)$ and $(d) \Rightarrow(e)$, nothing actually is needed a priori.

(ii) If we define the Hardy-Littlewood maximal operator, associated to $\Omega$,

$$
M_{\Omega} f(x)=\sup _{(y, t) \in \Omega_{x}} \frac{1}{|B(y, t)|} \int_{B(y, t)}|f(z)| d z,
$$

then (if $\Omega$ satisfies some mild conditions) $M_{\Omega} f \approx N_{\Omega}(|f|)$, and we thus obtain a characterization of the boundedness of $M_{\Omega}$ (see [NS] and [SS]).

(iii) As a consequence of the first remark and Proposition 2.5, it is easy to extend the characterization given in $[\mathrm{NS}]$, to atomic measures:

Corollary 2.8. Let $\nu$ be a positive, atomic and finite measure, and let $\Omega$ be an approach region satisfying the cone condition. Let $1 \leq p<\infty$. Then, the operator $N_{\Omega}: L_{\nu}^{p}\left(\mathbb{R}^{n}\right) \longrightarrow L^{p, \infty}\left(\mathbb{R}^{n}\right)$ is bounded if and only if $|\Omega(t)| \leq C t^{n}$, for all $t>0$. 


\section{EXAMPLE}

The following construction shows that there exists an approach region under the hypothesis of Theorem 2.6, larger than those considered in [NRS], for which the maximal operator is bounded (and hence, there exists almost everywhere convergence of the harmonic extension of functions in the corresponding potential space). We use the same ideas as in the example of a non-nontangential region in [NS]. Given $r$ as in the theorem, choose an increasing, $\mathcal{C}^{1}$ and convex function $\varphi:[0,1] \longrightarrow \mathbb{R}^{+}$, with $\varphi(0)=\varphi^{\prime}(0)=0, \varphi(1)<1$ and so that $t \geq \varphi(r(t))$ and $t / r(\varphi(t)) \longrightarrow \infty$ as $t \longrightarrow 0$. Define the sequences $t_{1}=\varphi(1), \ldots, t_{k}=\varphi\left(t_{k-1}\right)$, and $\left|y_{k}\right|=t_{k-1}$, with $\left|y_{1}\right|=1$. Finally, set

$$
\Omega=\bigcup_{k} \Gamma_{r}\left(y_{k}, t_{k}\right)
$$

Then $\Omega$ satisfies the $r$ condition, $\Gamma_{r} \subset \Omega$, for every $\alpha>0, \Omega \not \subset \Gamma_{\alpha r}$ and $|\Omega(t)| \leq$ $C r(t)^{n}$, for all $t>0$.

\section{REFERENCES}

[AC] M. Andersson and H. Carlsson, Boundary convergence in non-nontangential and nonadmissible approach regions, Math. Scand. 70 (1992), 293 -301. MR 93j:31008

[CS] M.J. Carro and J. Soria, Tent spaces over general approach regions and pointwise estimates, Pacific J. Math. 163 (1994), 217 -235. MR 95e:42012

[Li] J.E. Littlewood, On a theorem of Fatou, J. London Math. Soc. 2 (1927), 172 -176.

[NRS] A. Nagel, W. Rudin and J. Shapiro, Tangential boundary behavior of functions in Dirichlettype spaces, Ann. Math. 116 (1982), 331-360. MR 84a:31002

[NS] A. Nagel and E. Stein, On certain maximal functions and approach regions, Adv. Math. 54 (1984), 83-106. MR 86a:42026

[SS] A. Sánchez-Colomer and J. Soria, Weighted norm inequalities for general maximal operators and approach regions, Math. Nachr. 172 (1995), 249-260. MR 96e:42023

[St] E. Stein, Singular integrals and differentiability properties of functions, Princeton Univ. Press, 1970. MR 44:7280

Departamento Matemàtica Aplicada i Anàlisi, Universitat de Barcelona, 08071 BARCELONA, SpAin

E-mail address: raposo@cerber.mat.ub.es

E-mail address: soria@cerber.mat.ub.es 